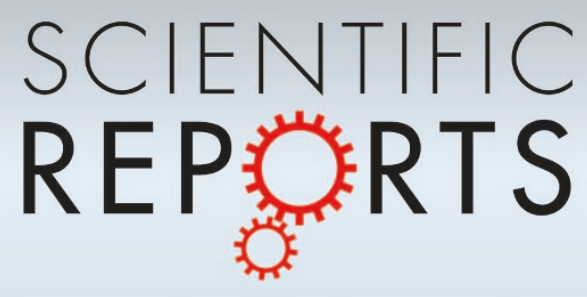

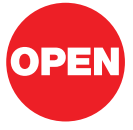

SUBJECT AREAS: OLFACTORY RECEPTORS

OLFACTORY BULB

FLUORESCENCE IMAGING

NEUROPHYSIOLOGY

Received

3 October 2012

Accepted

17 January 2013

Published

5 February 2013

Correspondence and requests for materials should be addressed to G.L. (loweg@monell.

org)

\section{Neural coding of binary mixtures in a structurally related odorant pair}

\author{
Georgina Cruz \& Graeme Lowe
}

Monell Chemical Senses Center, Philadelphia, Pennsylvania 19104-3308 U.S.A.

The encoding of odorant mixtures by olfactory sensory neurons depends on molecular interactions at peripheral receptors. However, the pharmacological basis of these interactions is not well defined. Both competitive and noncompetitive mechanisms of receptor binding and activation, or suppression, could contribute to coding. We studied this by analyzing responses of olfactory bulb glomeruli evoked by a pair of structurally related odorants, eugenol (EG) and methyl isoeugenol (MIEG). Fluorescence imaging in synaptopHluorin ( $\mathrm{spH}$ ) mice revealed that EG and MIEG evoked highly overlapped glomerular inputs, increasing the likelihood of mixture interactions. Glomerular responses to binary mixtures of EG and MIEG mostly showed hypoadditive interactions at intermediate and high odorant concentrations, with a few near threshold responses showing hyperadditivity. Dose-response profiles were well fitted by a model of two odorants competitively binding and activating a shared receptor linked to a non-linear transduction cascade. We saw no evidence of non-competitive mechanisms.

$\mathrm{n}$ both humans and animals, the response to an odorant mixture is generally not a linear sum of responses to individual components ${ }^{1-5}$. Deviations from additivity have been loosely termed 'mixture interactions', and include such diverse phenomena as suppression, masking, hyperadditivity (or synergy), hypoadditivity (or compression), configural perception, and overshadowing ${ }^{1,3,6-8}$. These interactions could arise at different stages in the olfactory sensory pathway, from initial events of odorant detection by the receptors of olfactory sensory neurons (OSNs) in the periphery ${ }^{9-11}$, to synaptic integration of odor encoding signals by central neural circuits $^{12-14}$. Peripheral mixture interactions may occur quite frequently because the combinatorial coding of odors by multiple, broadly tuned receptors means that different odorants are likely to be recognized by one or more shared receptors ${ }^{15,16}$.

What mechanisms underlie peripheral mixture interactions? Competitive binding and activation of shared receptors by different odorants acting as agonists could naturally lead to synergistic or hypoadditive interactions, through non-linear superposition of OSN responses by sigmoid dose-response curves ${ }^{17}$. On the other hand, studies of single OSN responses to odorant mixtures have described several other possible sources of interaction. Suppressive interactions may result from competitive antagonism by odorants that occupy binding sites without activating receptors ${ }^{18-21}$, or from non-competitive antagonism by parallel transduction systems ${ }^{22,23}$ and modulation of the ionic conductances generating OSN electrical responses ${ }^{24-26}$. The relative contributions of these, or other mechanisms to the peripheral neural coding of odorant mixtures is unclear. A recent survey of dosedependent responses of OSNs to binary odorant mixtures suggested that nearly half of all interactions depend on non-competitive mechanisms ${ }^{27}$.

Cataloging odorant responses of single OSNs is a labor intensive process that yields limited random samplings of mixture interactions from a large, diverse population of olfactory receptors. To acquire more systematic information about mixture interactions, we applied olfactory bulb (OB) glomerular imaging to analyze mixture responses of mouse olfactory receptors. Individual glomeruli receive sensory inputs from OSNs expressing unique receptors ${ }^{28}$, and their responses reveal interactions occurring at these receptors. This allowed us to test whether a competitive agonist model can account for mixture interactions of individual receptors of each glomerulus. We focused on binary mixtures of eugenol (EG) and methyl isoeugenol (MIEG). Choice of this odorant pair was motivated by their close structural similarity, which is expected to increase receptor overlap and interaction ${ }^{16}$, and by previous reports of antagonistic interactions at the receptor level ${ }^{21}$. Presynaptic glomerular responses to binary mixtures of varying ratios and concentrations were recorded in the dorsal OB of transgenic mice expressing a fluorescent activity reporter synaptopHluorin $(\mathrm{spH})$ in OSN terminals ${ }^{29}$. We found that many glomeruli exhibited dose-dependent responses to EG, MIEG, and to their binary mixtures. The extensive overlap of responsive glomeruli enabled us to analyze mixture interactions of multiple receptors over a range of stimulus concentrations. 


\section{Results}

EG and MIEG activate highly overlapped glomerular maps. The close molecular similarity of EG and MIEG (Fig. 1a) suggested that they would activate similar sets of glomeruli. We found that both EG and MIEG evoked responses in glomeruli occupying stereotypic positions in the dorsolateral $\mathrm{OB}$ in a rostrocaudal band, at relative positions consistent with previous reports ${ }^{30,31}$. Numbers of activated glomeruli varied with stimulus concentration. Fig. 1b compares MIEG glomerular activation maps over a range of concentrations $(0.1-50 \% \mathrm{v} / \mathrm{v}$ liquid dilution, and neat compound) to a reference EG map at fixed high concentration $(70 \% \mathrm{v} / \mathrm{v})$. Overlap of MIEG and EG maps was the smallest at lowest concentration, and progressively increased at higher MIEG concentrations as more glomeruli were recruited. At the highest concentration, a single glomerulus (white arrow) responding to both odorants accounted for the differences. A similar progression was observed in reciprocal comparisons between EG maps at varying concentration and a reference MIEG map at 70\% $\mathrm{v} / \mathrm{v}$ (Fig. 1c). These trends were quantified by computing correlation coefficients pixel-wise from binary thresholded intensity maps (Fig. 1d). High correlation $(r>0.7)$ over a wide concentration range confirmed that molecular similarity of EG and MIEG is recapitulated in strong overlap of glomerular maps. This strong overlap was maintained independent of variability in locations of responsive glomeruli between animals ${ }^{30,32}$. Our results agree with previous findings of maps having strong overlap $(r>0.5)$ for similar odorant pairs, and weak overlap for dissimilar pairs $(\mathrm{r}<0.5)^{33}$.

EG and MIEG responses have similar kinetics but different dose-response relations. Both EG and MIEG evoked slow transient elevations in glomerular fluorescence lasting many seconds, similar to $\mathrm{spH}$ responses reported previously for other odorants ${ }^{29}$. Figure $2 \mathrm{a}$ shows typical rising phases and peaks of responses of a single glomerulus to 3 non-consecutive trials of EG stimulation at the same concentration. We selected 18 glomeruli from 4 mice for detailed analysis of response time courses. At higher stimulus concentrations $(>0.1-1 \% \mathrm{v} / \mathrm{v})$, inter-trial variation in EG and MIEG responses was $\sim 10-20 \%$ for peak amplitudes (mean \pm SD, $16.9 \pm 2.5 \%$ ) and rise-times (mean \pm SD $16.9 \pm 3.6 \%$ ). Responses of the same glomerulus to different odorants or stimulus concentrations followed similar time courses that could be matched by scaling (Figs. 2b,c). In Fig. 2b, rescaling EG responses at intermediate concentration $(0.53 \mathrm{ppm})$ to match those at high concentration (2.21 ppm) yielded time courses that were not significantly different ( $t$-test, $P>0.05)$. In Fig. $2 c$, maximal responses to high concentrations of EG (1.68 ppm) and MIEG (4.65 ppm) were also matched by rescaling $(\mathrm{P}>0.05)$. Among 36 rescaling tests of similarity of responses to the same odorant, 29 were matched $(\mathrm{P}>0.05)$. In addition, 13 of 18 rescaling tests of responses to different odorants (EG vs. MIEG) were matched $(\mathrm{P}>0.05)$. The few cases with significant differences occurred when there was high noise in weak responses, or responses had multiple peaks (e.g. black trace, Fig. 2a). Invariance of time course between EG and MIEG over a range of stimulus concentrations agrees with previous reports that $\mathrm{spH}$ response kinetics are similar for different odorants and response amplitudes ${ }^{29,34}$.

Maximal amplitudes of EG or MIEG responses were variable across different glomeruli and animals, as expected from diversity of olfactory receptors and experimental variability in background fluorescence. However, maximal responses evoked by neat odorants tended to be smaller for EG than MIEG. In pair-wise comparisons of maximal EG and MIEG responses of the 18 glomeruli above, EG response exceeded MIEG response in only $2 / 18$ glomeruli, and responses were approximately equal in 4/18 glomeruli. Values of EG-toMIEG scale factors in cases where the MIEG response was nearly the same as or larger than the EG response were 0.97-1.52 (1.17 \pm 0.17 , mean $\pm \mathrm{SD}, n=12$ rescalings in 8 glomeruli). Thus, the maximal EG response can be as much as 50\% less than the maximal MIEG response. This is consistent with a previous study that used calcium imaging to measure dose responses of several glomeruli in the dorsal olfactory bulb and found higher average sensitivity to MIEG than to $\mathrm{EG}^{30}$.

Glomerular responses to EG and MIEG were recorded over 2 log units of stimulus concentration (Fig. 2d). Previously, dose-response relations were analyzed by describing the entire response, from odorant binding at receptors to glomerular activation, by a simple Hill equation $^{29,35}$. Here we introduce a more detailed description that separates odorant binding and receptor activation steps from the cooperative olfactory transduction cascade. This enabled us to develop a new, self-consistent model of mixture interactions (see Supplementary Information). Dose-response relations for single odorants were plotted as peak response amplitude, $F$, vs. stimulus concentration, $X$, and fitted to the function:

$$
F=F_{1 D}(X)=\frac{F_{\max }}{1+\frac{1}{\eta^{n_{\mathrm{H}}}}\left(1+\frac{K}{X}\right)^{n_{\mathrm{H}}}}
$$

This formula is based on an equilibrium model in which an odorant binds non-cooperatively to an olfactory receptor at a single binding site, leading to single-step receptor activation ${ }^{27,36}$. The activated odorant-receptor complex triggers a sequence of transduction steps: G-protein-coupled activation of adenylyl cyclase ${ }^{37,38}$, synthesis of cyclic $\mathrm{AMP}^{39}$, cooperative gating of cationic and chloride channels ${ }^{40}$, action potential firing and ultimately transmitter release at OSN terminals reported by the $\mathrm{spH}$ signal. Fitted parameters were: $F_{\max }$, upper limit of the response with transduction fully activated; $K$, odorant affinity for the receptor, i.e. odorant concentration for halfmaximal receptor activation; $n_{\mathrm{H}}$, a Hill coefficient describing the collective cooperativity of all transduction steps; and $\eta=\mathrm{R}^{*} / K^{*}$, a measure of the efficiency of activation of the transduction steps, equal to the ratio of $\mathrm{R}^{*}$ (= maximal achievable concentration of odorant-receptor complex), to $K^{*}$ (= concentration of odorantreceptor complexes needed for $50 \%$ activation of downstream transduction). The value of $\eta$ is set by the total pool of receptors available for odorant binding and activation, i.e. the spare receptor capacity ${ }^{41}$. A large capacity (high $\eta$ ) allows more complete activation of transduction at saturating odor concentrations, bringing the response closer to the upper limit.

Single odorant dose-response relations were well fitted by Eqn (1). Dynamic ranges spanned at least two log units, with saturation at the highest odorant concentrations (Figs. $2 \mathrm{~b}$ and d). Data were acquired for 3-6 glomeruli per mouse, and divided into two groups: (i) highly sensitive glomeruli $(n=4)$ whose responses were already saturated with nearly flat dose-response curves over $2 \log$ units, and activation thresholds $<0.3 \mathrm{ppm}$ (lowest tested concentration); (ii) moderately sensitive glomeruli $(n=15)$, with graded responses saturating at higher concentrations. Amplitudes of saturated responses were variable within each group. Only the moderately sensitive glomeruli could be fitted to Eqn (1). Fig. 2d shows fits to EG dose-response data from 4 of these glomeruli in the same mouse, with different dose-response profiles and parameters.

Fit parameters were extracted from dose-response relations of all 15 overlap glomeruli activated by both EG and MIEG. Their distributions are plotted in Figs. 2e-g, and their variation summarized in Table 1. Overlap glomeruli exhibited a range of apparent affinities for EG and MIEG (Fig. 2e), consistent with the diversity of olfactory receptors mapped to different glomeruli. Transduction cooperativity $\left(n_{\mathrm{H}}\right)$ was strongly positive and not significantly different for the two odorants $\left(n_{\mathrm{H} \mathrm{EG}}=3.86 \pm 1.12, n_{\mathrm{H} \text { MIEG }}=3.68 \pm 1.03 ; \mathrm{P}=0.23\right.$, paired, 2 tailed t-test, $n=30$ ). We found ca. 3-fold variation of cooperativity across glomeruli $\left(n_{\mathrm{H}}=2.08-6.36\right)$, similar to ca. 4 -fold variation (0.5-1.9) reported in calcium imaging measurements ${ }^{35}$. Our higher $n_{\mathrm{H}}$ values may come from separate modeling of 
a

Eugenol (EG)<smiles>C=CCc1ccc(O)c(OC)c1</smiles>

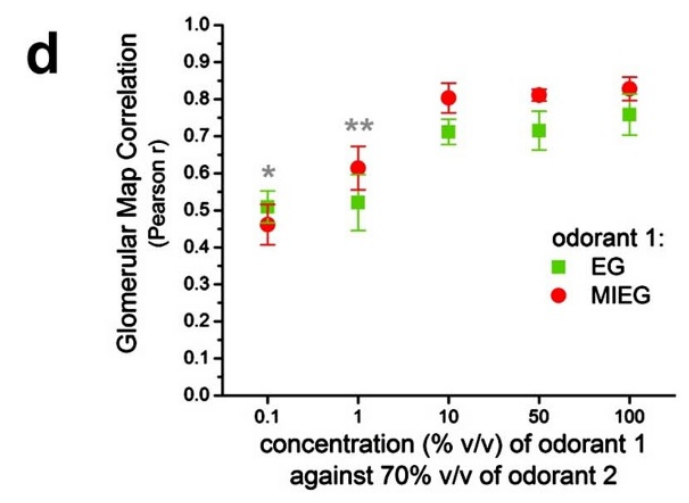

C

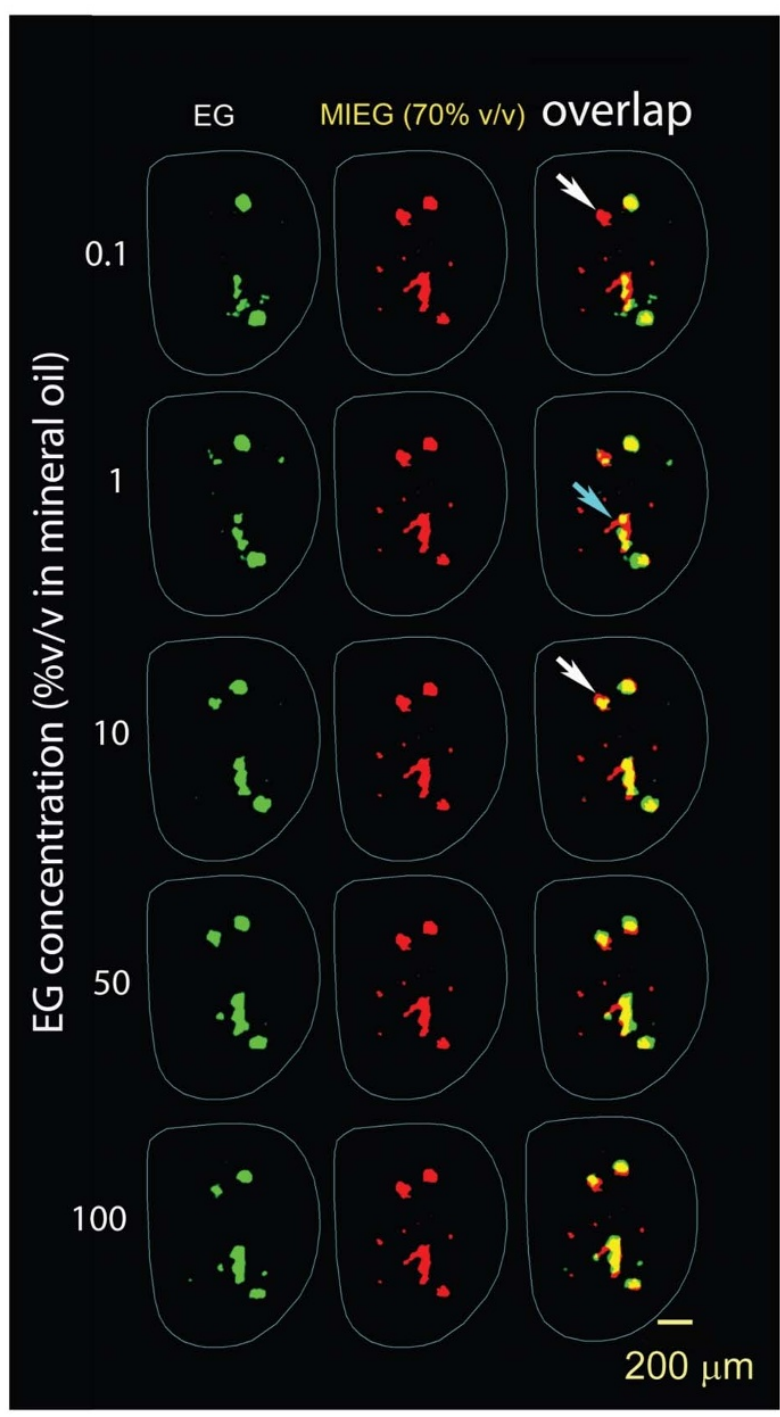

Figure 1 Eugenol (EG) and methyl isoeugenol (MIEG) evoke highly overlapped maps of glomerular activity in the mouse olfactory bulb. (a). Chemical structures and physicochemical properties of odorants EG and MIEG. (b). Overlap of glomerular activity patterns evoked by varying concentrations of MIEG vs. a fixed reference concentration of EG. Color-coded intensity plots show spatial distributions of synaptopHluorin (spH) fluorescence. Left column: red color-coded maps of the dose-dependent response to MIEG over 0.1, 1, 10, 50\% v/v liquid dilution in mineral oil, $100 \%$ or neat odorant; middle column: green color-coded map of the response to EG at fixed concentration (70\% v/v); right column, merged red and green maps, showing increasing overlap (yellow) of glomerular activation patterns with increasing MIEG concentration. White arrows: a glomerulus shared by both odorants only at relatively high odorant doses. Cyan arrows: a glomerulus activated by MIEG, but not by EG, at high concentration, considered to be a non-specific response. (c). Reciprocal data acquired from the same animal on a different day with MIEG and EG reversed (d). Summary of overlap correlations between EG and MIEG glomerular maps in reciprocal experiments. A binary threshold was applied to the maps in (b) and (c), and correlation coefficients (Pearson's r) were calculated pixel-by-pixel for the series of MIEG maps against a fixed 70\% v/v EG map as in (b) (red circles), and vice versa (green squares). Asterisks indicate significant differences between overlapped maps at lower doses of 0.1 and $1 \% \mathrm{v} / \mathrm{v}$ and those at the higher dose levels of $10-100 \% \mathrm{v} / \mathrm{v}$ for both reciprocal experiments (one-way ANOVA and Tukey's test, $\mathrm{P}<0.05, \mathrm{n}=3$ mice). Population variances were not significantly different between tested groups (Levene's test; $\mathrm{EG}+\mathrm{MIEG}$ : $\mathrm{P}=0.665$, MIEG +EG: $\mathrm{P}=0.362$ ). 
a

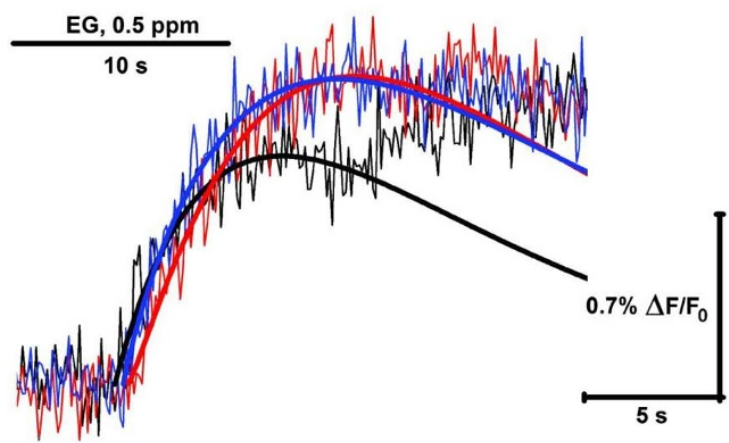

C

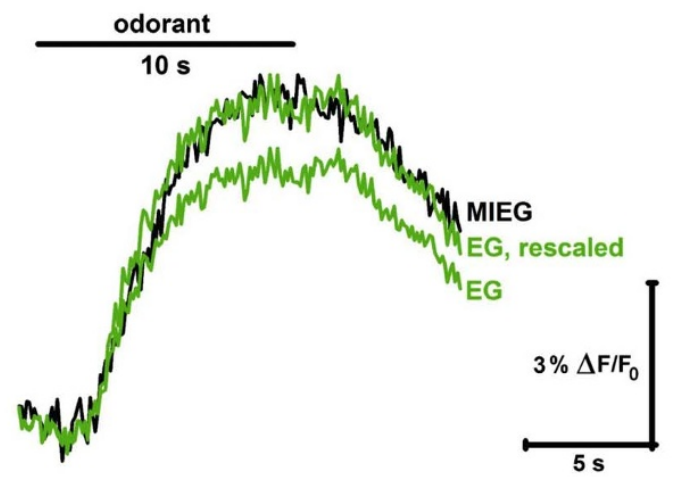

b

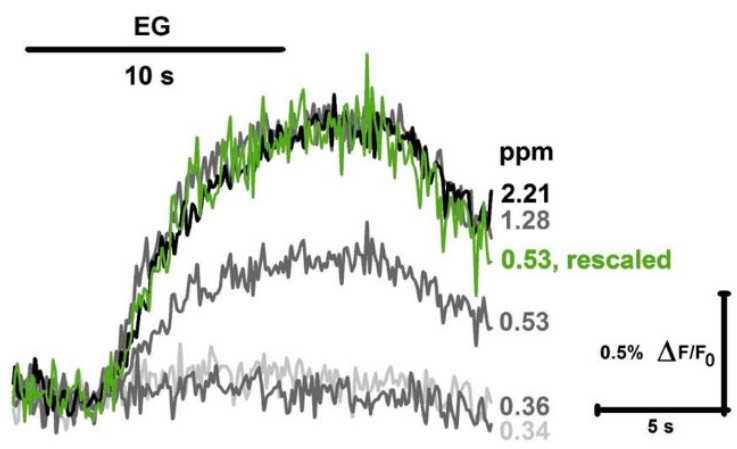

d

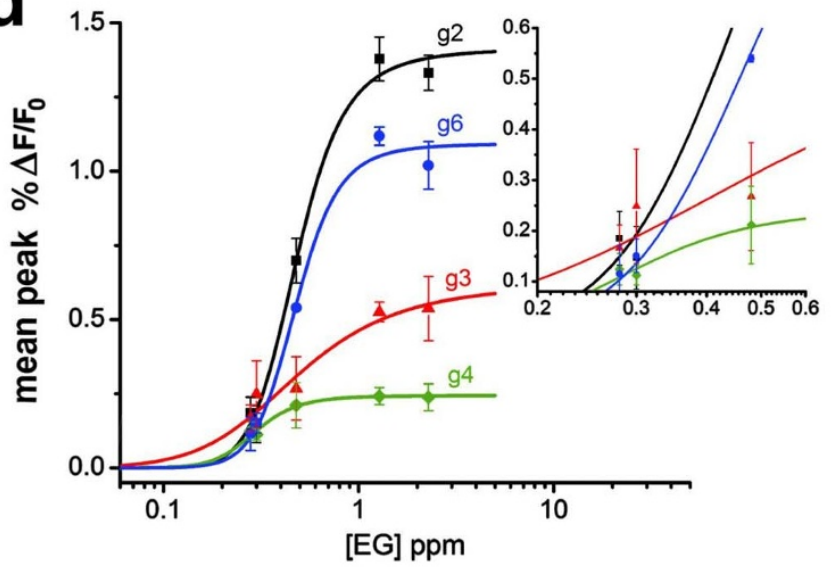

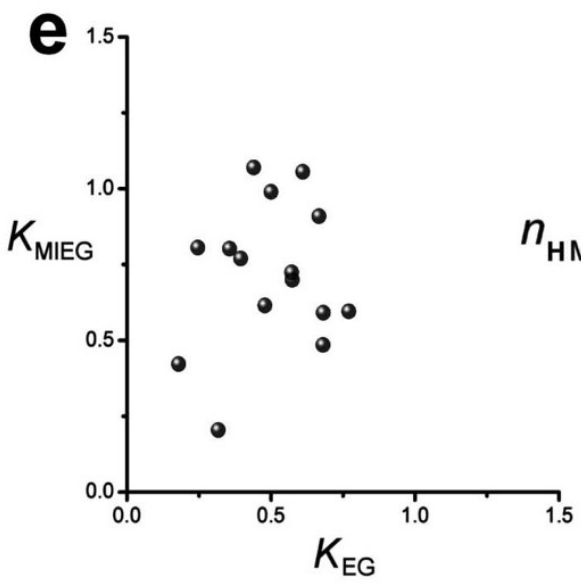
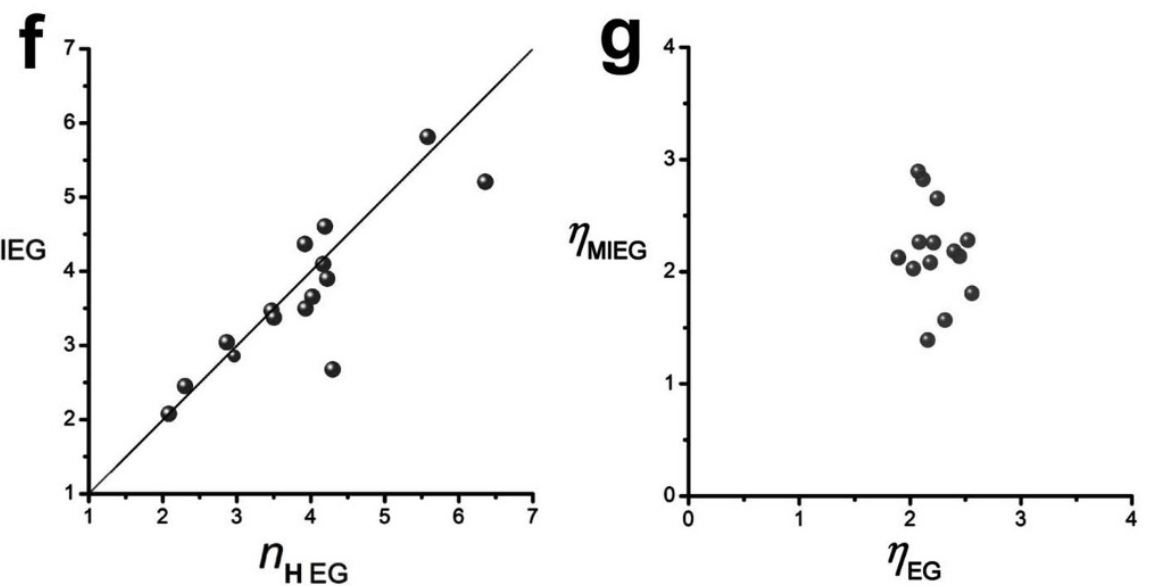

Figure $2 \mid$ Dynamics and concentration dependence of EG- and MIEG-evoked spH fluorescence responses in individual glomeruli. (a). Raw traces showing inter-trial variation of the rising phases and peaks of the relative change, $\Delta \mathrm{F} / \mathrm{F}_{0}$, in spH fluorescence of a glomerulus evoked by repeated, semirandomized odorant stimuli (EG, $0.5 \mathrm{ppm}$ ). Alpha function curve fits to the data (see Methods) are overlaid for each raw data trace. Horizontal bar shows timing and duration of odorant pulses. (b). Dose-dependence of $\Delta \mathrm{F} / \mathrm{F}_{0}$ responses of another glomerulus to EG. Gray traces show responses to stimuli delivered at ascending concentrations as indicated to the right of each trace, with saturation occurring at $>1.28$ ppm. Time courses of responses were concentration invariant at intermediate and higher doses, as shown by overlay of a rescaled $0.53 \mathrm{ppm}$ response (green trace) and maximum $2.21 \mathrm{ppm}$ response. Similar data were obtained for responses to MIEG. (c). Odorant stimulus invariance of the time courses of $\Delta \mathrm{F} / \mathrm{F}_{0}$ responses of a third glomerulus to EG (1.68 ppm, green traces) and MIEG (4.65 ppm, black trace). Upper green trace shows how the time course of the EG response could be rescaled to match the time course of the MIEG response. Horizontal bars in (a-c) show timing and duration of odorant pulses. Rescaling factors in (b) and (c) were obtained by minimizing areas between responses. (d). Dose-response profiles of peak EG responses of four glomeruli (g2, g3, g4, g6) from the same animal, recorded in the same experiment. Plotted points represent values averaged over 3 trials (mean \pm SD). Fitted curves were obtained from a modified Hill equation (see Eqn (1) in text) with parameters $\left(F_{\max }, K, n_{\mathrm{H}}, \eta\right)$ values of: g2 $(1.43,0.49,6.82,2.01)$; g3 $(0.73,0.52,2.74,1.85)$; g4 (0.24, 0.49, 6.31, 2.64); g6 $(1.09,0.96,5.86,3.01)$. Inset: magnified view near threshold highlighting the variability in fits for different glomeruli. (e-g). Scatter plots of values of the parameters $K, n_{\mathrm{H}}, \eta$ obtained from 15 glomeruli by fitting dose-response relations for odorants EG and MIEG to Eqn (1). Line in (f) indicates diagonal axis. 
Table 1 | Variation in model parameters obtained by fitting single odorant dose-response relations of 15 individual glomeruli to a modified Hill equation (Eqn (1) in text) (data from 4 mice). RSS (residual sum of squares) is the fitting error

\begin{tabular}{|c|c|c|c|c|c|c|}
\hline & \multicolumn{3}{|c|}{ EG } & \multicolumn{3}{|c|}{ MIEG } \\
\hline & range & median & mean $\pm S D$ & range & median & mean $\pm S D$ \\
\hline$K$ [ppm] & $0.178-0.769$ & 0.498 & $0.496 \pm 0.174$ & $0.206-1.069$ & 0.725 & $0.716 \pm 0.241$ \\
\hline$F_{\max }$ & $0.306-1.452$ & 0.998 & $0.879 \pm 0.342$ & $0.508-2.044$ & 0.921 & $1.004 \pm 0.456$ \\
\hline$n_{H}$ & $2.084-6.360$ & 3.932 & $3.861 \pm 1.116$ & $2.087-5.817$ & 3.504 & $3.679 \pm 1.026$ \\
\hline$\eta$ & $1.890-2.552$ & 2.177 & $2.203 \pm 0.206$ & $1.387-2.889$ & 2.134 & $2.171 \pm 0.409$ \\
\hline RSS & $0.022-0.372$ & 0.138 & $0.149 \pm 0.089$ & $0.083-0.679$ & 0.234 & $0.271 \pm 0.171$ \\
\hline
\end{tabular}

transduction cooperativity in Eqn (1), which would reveal the high cooperativity of downstream transduction steps $\mathrm{s}^{40,42}$. The values were well correlated between EG and MIEG (Fig. 2f; Pearson's correlation coefficient $\mathrm{r}=0.9907, n=15)$. Efficiencies of activation $(\eta)$ were not significantly different between EG and MIEG $\left(\eta_{\mathrm{EG}}=2.2 \pm 0.21\right.$; $\eta_{\text {MIEG }}=2.17 \pm 0.41 ; \mathrm{P}=0.80$, paired, 2 tailed t-test, $\left.n=30\right)$ and both were close to $\eta=2$ (Fig. 2g). This implies that $K_{\mathrm{EG}}$ and $K_{\mathrm{MIEG}}$ may be interpreted as nominal concentrations for half-maximal response (Supplementary Information). The estimated upper limit of responses $\left(F_{\max }\right)$ was significantly higher for MIEG than EG ( $F_{\max }$ EG $=1.45<F_{\max }$ MIEG $=2.04$ ), consistent with the distribution of MIEG-to-EG scale factors for response time courses.

EG and MIEG exhibit binary mixture interactions. To analyze odorant mixture interactions, we characterized dose-dependent responses of glomeruli to binary mixtures of EG and MIEG. Considering that MIEG can antagonistically modulate EG responses of some olfactory receptors ${ }^{21}$, we designed reciprocal experiments to systematically examine modulatory effects of MIEG on EG responses, and vice versa. First, we applied 5 concentrations of EG alone as test odorant, then 15 binary mixtures of EG with MIEG added in various ratios as modulating odorant, and then 3 concentrations of MIEG alone. Next, we applied the reciprocal protocol to the same mouse, with the odorants interchanged (MIEG test, EG added). Figs. 3a-d show reciprocal families of binary mixture dose-response curves for two representative glomeruli, one with high EG/MIEG sensitivity (3a, 3b), the other with moderate sensitivity (3c, 3d). In the high sensitivity glomerulus, responses to the test odorant (black plots) were already saturated (flat curves), while in the moderate sensitivity glomerulus they increased with concentration (curves with positive slope). When ascending concentrations of added odorant were mixed with test odorant (blue, green and red plots), we observed no significant elevations of responses in the high sensitivity glomerulus, and incremental elevations in the moderate sensitivity glomerulus. In neither case did added odorant antagonize responses to test odorant. In some high sensitivity glomeruli, there was a slight reduction in response when the highest concentration stimuli were applied separately or in mixtures near the end of each recording session (Figs. 3a, 3b). We attributed this effect to adaptation or desensitization rather than antagonism, because it was seen for both test and added odorant, and the highest concentrations of added odorant did not cause greater reductions in the responses to the lowest concentrations of test odorant. Comparing responses to binary mixtures and single added odorant (left-most points, 3c, 3d), approximate additivity was seen at lower doses, and hypoadditivity at higher doses. We compared mixture responses to the algebraic sum of EG and MIEG responses at high (50\% test $+70 \%$ added component) and low $(0.1 \%$ or $1 \%$ test $+10 \%$ added component) ends of our concentration range. In all 15 overlap glomeruli examined, we found significant non-additivity of responses, but no examples of antagonism. In 51 comparisons of mixture and summed responses of these glomeruli (3 repeated trials each), there were 35 cases of hypoadditivity (24 high concentration, 11 low), 13 cases of additivity ( 1 high,
12 low), and 3 cases of hyperadditivity (low concentration) $(\mathrm{P}<0.05$, Kolmogorov-Smirnov tests).

If mixture interactions are defined as deviations from additivity, then glomerular responses to EG and MIEG clearly exhibited such interactions at both low and high doses. In general, we may expect similar mixture interactions for any pair of stimuli that share a nonlinear, sigmoidal dose-response relation like Eqn (1). The nonlinearity can arise from cooperativity of transduction at low odorant doses, and from response compression by saturation of odorant binding, receptor activation and transduction at high doses. Since EG and MIEG have similar molecular structures, their mixture interactions may depend entirely on competitive binding to olfactory receptors, and may be described by the nonlinearity in Eqn (1). To test this hypothesis, we fitted our binary mixture dose-response data to a glomerular response model that incorporates the pharmacology of competitive receptor binding and activation. We assumed that two odorants (EG and MIEG) compete for the same receptor binding site in the population of OSNs projecting to a given glomerulus. As in Eqn (1), ligand binding is followed by single-step receptor activation and the triggering of a cooperative series of transduction steps. Each odorant is now permitted to have its own independent binding affinity $K$, and efficiency of transduction activation $\eta$, although a common Hill coefficient $n_{\mathrm{H}}$ still describes cooperativity of the shared transduction cascade. This leads to a formula that is a 2 -dimensional (2D) extension of Eqn (1) (Supplementary Information). The peak response amplitude, $F$, is a function of two variables $(U, V)$, representing concentrations of each odorant (i.e., [EG], [MIEG]) in binary mixtures:

$$
F=F_{2 \mathrm{D}}(U, V)=\frac{F_{\max }}{1+\left[\frac{K^{*}}{R^{*}(U, V)}\right]^{n_{\mathrm{H}}}}=\frac{F_{\max }}{1+\left[\frac{1+\frac{U}{K_{\mathrm{U}}}+\frac{V}{K_{\mathrm{V}}}}{\eta_{U} \frac{U}{K_{\mathrm{U}}}+\eta_{V} \frac{V}{K_{\mathrm{V}}}}\right]^{n_{\mathrm{H}}}}
$$

with: $n_{\mathrm{H}}$ the shared cooperativity, $R^{*}(U, V)$ the concentration of activated ligand-receptor complex, $K^{*}$ the activation constant of transduction, $\left(K_{U}, K_{V}\right)$ the receptor binding and activation constants for each odorant, and $\left(\eta_{\mathrm{U}}, \eta_{\mathrm{V}}\right)$ the efficiencies of transduction activation for each odorant. This model provided good fits to mixture responses of moderate sensitivity glomeruli (Figs. 3c, 3d). Inspection of parameter values obtained from $2 \mathrm{D}$ fits to 30 reciprocal experiments on 15 glomeruli revealed similar values to those obtained from 1D fits to single-odorant dose-responses (Figs. 3e, 3f, 4a-e, Table 2). Comparisons of $1 \mathrm{D}$ and $2 \mathrm{D}$ fit results revealed that population medians of each parameter did not differ significantly between groups (Kruskal-Wallis ANOVA; $\mathrm{P}=0.88\left(K_{\mathrm{U}}, K_{\mathrm{V}}\right), 0.44$ $\left.\left(\eta_{\mathrm{U}}\right), 0.48\left(\eta_{\mathrm{V}}\right), 0.65\left(n_{\mathrm{H}}\right), n=30\right)$. This indicated that responses to binary mixtures were consistent with, and predictable from, responses to each mixture component. To test the accuracy of parameter estimates, we applied our fitting routine to 8 mock data sets generated from Eqn (2) (see Methods). In all cases we successfully recovered input parameters within statistical error of fit (Supplementary Information, Tables ST1, ST2). We therefore concluded 

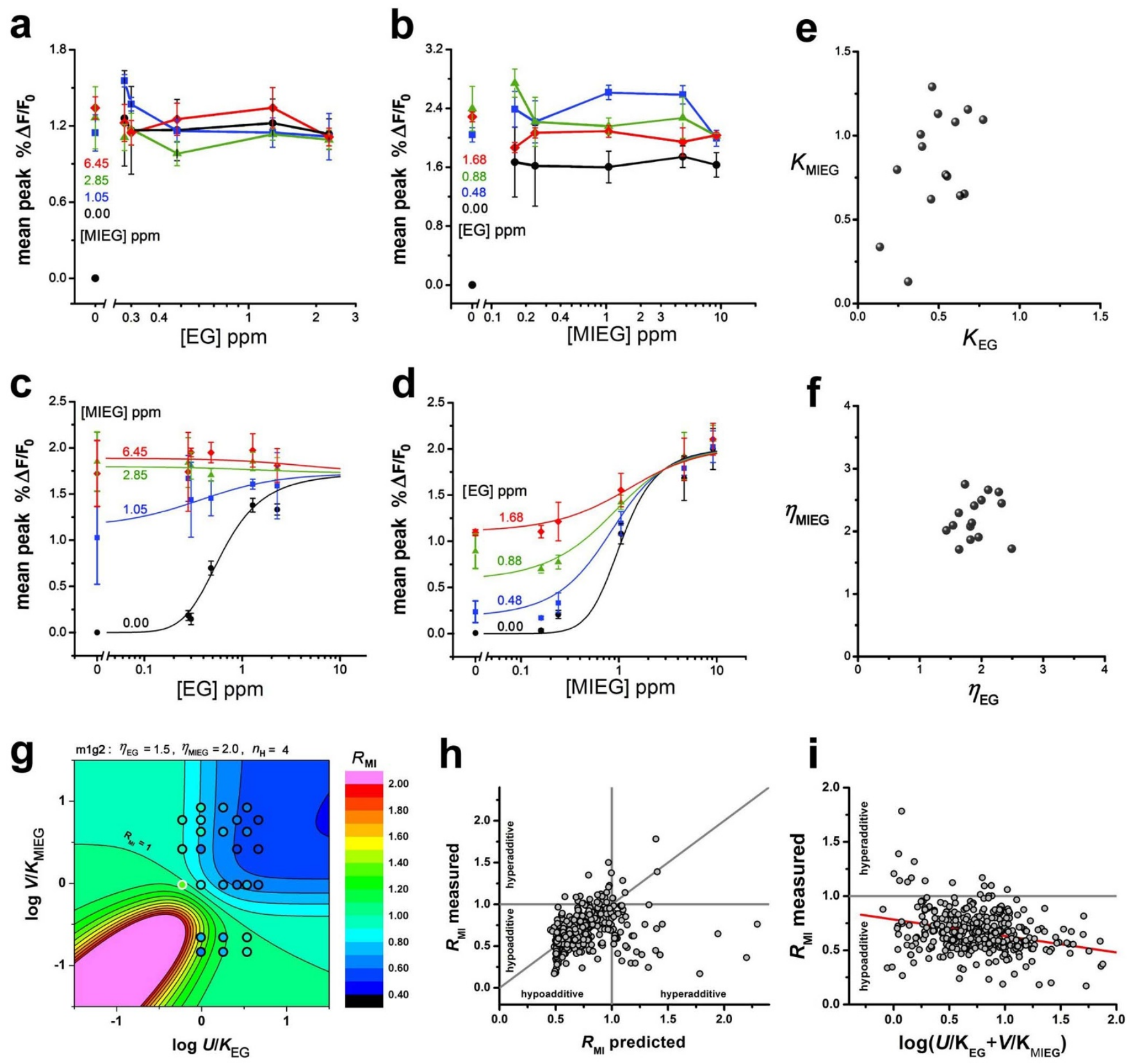

Figure 3 Glomerular dose-responses and model fits for binary mixture interactions. (a). Dose-response plots of high sensitivity glomerulus for EG (black), and EG with added MIEG (blue, green and red). (b). Reciprocal plots for same glomerulus as in 3a, for MIEG (black), and MIEG with added EG (blue, green and red). (c). Dose-response plots of moderate sensitivity glomerulus for EG (black), and EG with added MIEG (blue, green and red). Fitted curves correspond to $2 \mathrm{D}$ model parameters: $F_{\max }=2.01 ; K_{\mathrm{EG}}=0.49 ; K_{\mathrm{MIEG}}=1.04 ; n_{\mathrm{H}}=4.17 ; \eta_{\mathrm{EG}}=1.63 ; \eta_{\mathrm{MIEG}}=2.18$. (d). Reciprocal plots for the same glomerulus as in 3c, for MIEG (black points) and MIEG with added EG (blue, green and red). Fitted curves correspond to 2D model parameters: $F_{\max }=2.12 ; K_{\mathrm{EG}}=0.71 ; K_{\mathrm{MIEG}}=1.13 ; n_{\mathrm{H}}=4.47 ; \eta_{\mathrm{EG}}=1.45 ; \eta_{\mathrm{MIEG}}=2.01$. In a-d, responses to added odorant alone are plotted on the left. Plotted points are averages over 3 trials (mean $\pm \mathrm{SD}$ ). (e, f). Plots of model parameter values $K$ and $\eta$, obtained from 15 glomeruli by $2 \mathrm{D}$ model fits to EG and MIEG responses. (g). Contour plot of theoretical mixture interaction ratio, $R_{\mathrm{MI}}$, as a function of $U=[\mathrm{EG}], V=[\mathrm{MIEG}]$ (log scales normalized to $K$ values) calculated by Eqn (3) for: $n_{\mathrm{H}}=4, \eta_{\mathrm{EG}}=1.5, \eta_{\mathrm{MIEG}}=2.0$ (parameters from 2D fits for glomerulus m1g2, same as (3c-d). Boundary contour $R_{\mathrm{MI}}=1$ separates hypoadditive (upper right) and hyperadditive (lower left) regions. Experimental ratios are overlaid as circles (same color scale as contour plot) to compare model and experimental values. Black circle border: hypoadditive responses; white border: hyperadditive response. (h). Plot comparing experimental and theoretically predicted $R_{\mathrm{MI}}$ for 450 mixture responses of 15 moderate sensitivity glomeruli. Predicted values are from Eqn (3) with model parameters obtained by $2 \mathrm{D}$ fitting of mixture dose-responses of individual glomeruli. (i). Plot of same experimental $R_{\mathrm{MI}}$ values against log of summed normalized odorant mixture concentrations (15 glomeruli). Red line is linear regression showing inverse correlation $(\mathrm{r}=-0.2953, \mathrm{P}<0.0001)$.

that, for all tested glomeruli, mixture interactions of EG and MIEG were well described by the competitive agonist model.

To more clearly visualize mixture interactions and their predictability from our model, we quantified the degree of interaction by computing $R_{\mathrm{MI}}$, the ratio of the mixture response to the sum of individual responses to each odorant. This serves as an empirical index of deviation from linear additivity of responses. In our model, the theoretical value of $R_{\mathrm{MI}}$ is: 

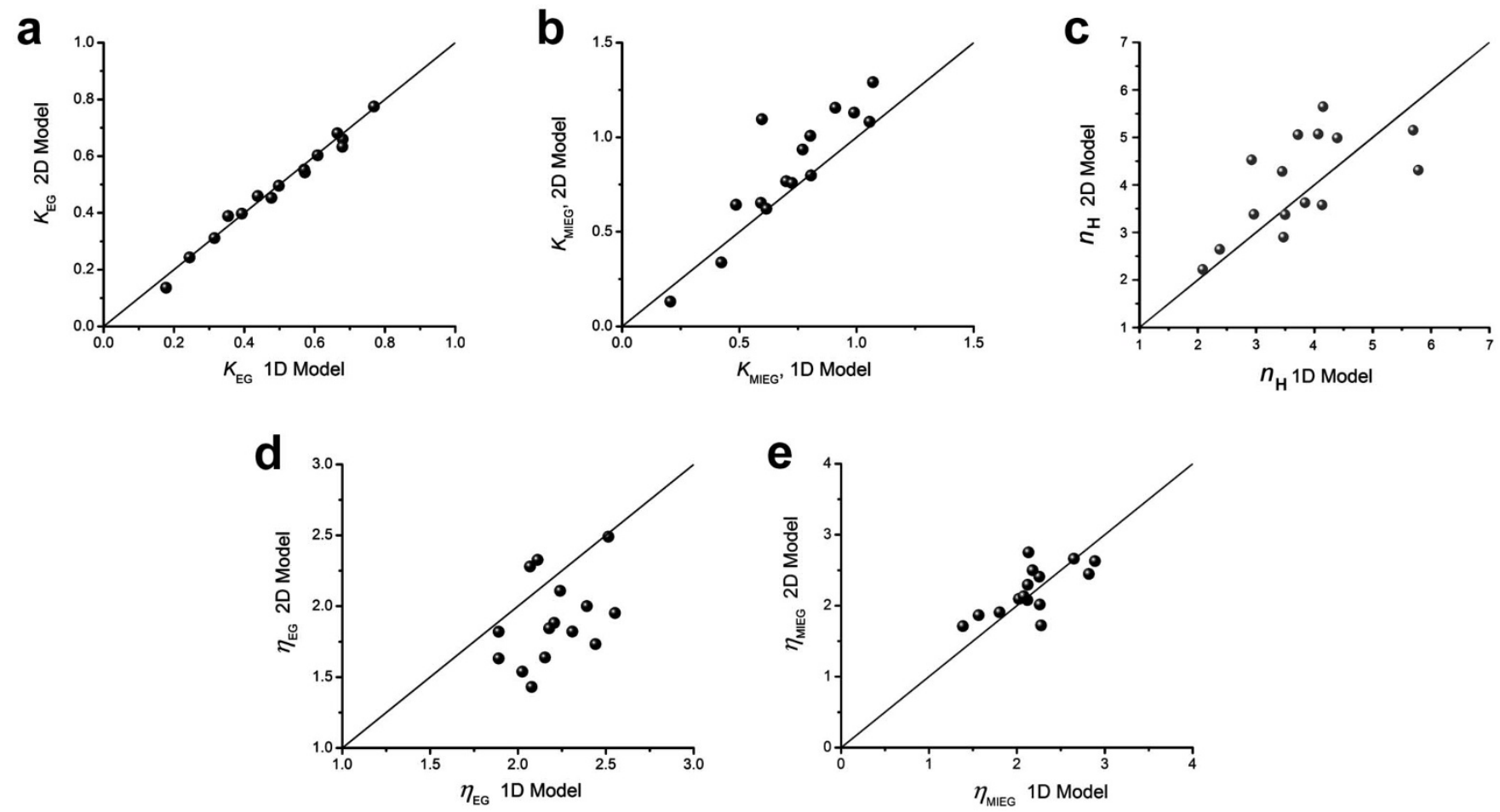

Figure $4 \mid$ Comparison of parameters from 1D (single odorant) and 2D (binary mixture) model fits. Scatter plots of 2D vs. 1D parameters:

(a) $K_{\mathrm{EG}}$; (b). $K_{\mathrm{MIEG}}$; (c). $n_{\mathrm{H}}$; (d). $\eta_{\mathrm{EG}}$; (e). $\eta_{\mathrm{MIEG}}$. The $1 \mathrm{D}$ values were obtained from fits of receptor binding, activation and transduction model, Eqn (1), to single odorant dose response data; the $2 \mathrm{D}$ values from fits of competitive binary interaction model, Eqn (2), to mixture dose response data.

Diagonal reference lines are drawn to show how well $1 \mathrm{D}$ values predicted $2 \mathrm{D}$ values. Linear regression yielded significant correlations between $1 \mathrm{D}$ and $2 \mathrm{D}$ values except for $\eta_{\mathrm{EG}}$, which had small overall variation, with values closely clustered around $\eta_{\mathrm{EG}}=2$. Pearson's correlation coefficients were: $K_{\mathrm{EG}}$, $\mathrm{r}=0.9905, \mathrm{P}<0.001 ; K_{\mathrm{MIEG}}, \mathrm{r}=0.8968, \mathrm{P}<0.001 ; n_{\mathrm{H}}, \mathrm{r}=0.6379, \mathrm{P}=0.0105 ; \eta_{\mathrm{EG}}, \mathrm{r}=0.3874, \mathrm{P}=0.1537 ; \eta_{\mathrm{MIEG}}, \mathrm{r}=0.6844, \mathrm{P}=0.0049$.

$$
R_{\mathrm{MI}}(U, V)=F_{2 \mathrm{D}}(U, V) /\left\{F_{2 \mathrm{D}}(U, 0)+F_{2 \mathrm{D}}(0, V)\right\}
$$

We created $2 \mathrm{D}$ color plots in which experimental $R_{\mathrm{MI}}$ values calculated from the data, were overlaid onto contour plots of $R_{\mathrm{MI}}(U, V)$ calculated from fitted parameters (Fig. $3 \mathrm{~g}$ ). Using logarithmic axes normalized to odorant affinity, the mixture concentration plane $(U, V)$ was divided into two continuous regions according to the theoretical value of $R_{\mathrm{MI}}(U, V)$ : (i) $R_{\mathrm{MI}}>1$, a region of lower concentration and hyperadditive responses (synergy) due to positive cooperativity; and (ii) $R_{\mathrm{MI}}<1$, a region of higher odorant concentration and hypoadditive responses (compression) due to response saturation. These regions are separated by a boundary $\left(R_{\mathrm{MI}}=1\right)$ along which the responses are exactly additive. This topology is a general property of two variable, double sigmoid functions described by Eqn (2). In Fig. 3h, $R_{\mathrm{MI}}$ values of data pooled from 15 glomeruli are plotted against values theoretically predicted from model fits to each glomerulus. Hypoadditive interactions were fairly well predicted by the model $(\mathrm{r}=0.5772, \mathrm{P}<0.0001 ; n=381$ points in lower left quadrant). Thus, the nonlinear properties of the competitive model (Eqn (2)) was a good predictor of observed mixture interactions. Errors in predicted $R_{\mathrm{MI}}$ for hyperadditive interactions (lower right quadrant of Fig. $3 \mathrm{~h}$ ) might be due to the sparseness of our measurements of cooperative responses near threshold. Plotting $R_{\mathrm{MI}}$ versus total normalized mixture concentration confirmed that hypoadditivity occurred predominantly at higher concentrations, and the few examples of hyperadditivity occurred at lower concentrations (Fig. 3i).

\section{Discussion}

We measured the activation of OSN presynaptic terminals by EG and MIEG on the dorsal surface of the olfactory bulb and constructed dose-response curves over 2 log units of stimulus concentration. As EG and MIEG share similar molecular structures, there was a large overlap in their glomerular maps. Binary mixture responses in overlap glomeruli all exhibited deviations from linear additivity (mixture interactions). The dominant interaction was hypoadditivity at intermediate and high odorant doses, although there were a few examples of hyperadditivity at low doses. These findings are consistent with

Table 2 | Variation in model parameters obtained by fitting binary odorant mixture dose-response relations of 15 individual glomeruli to a competitive binding model equation (Eqn (2) in text) (data from 4 mice). RSS (residual sum of squares) is the fitting error

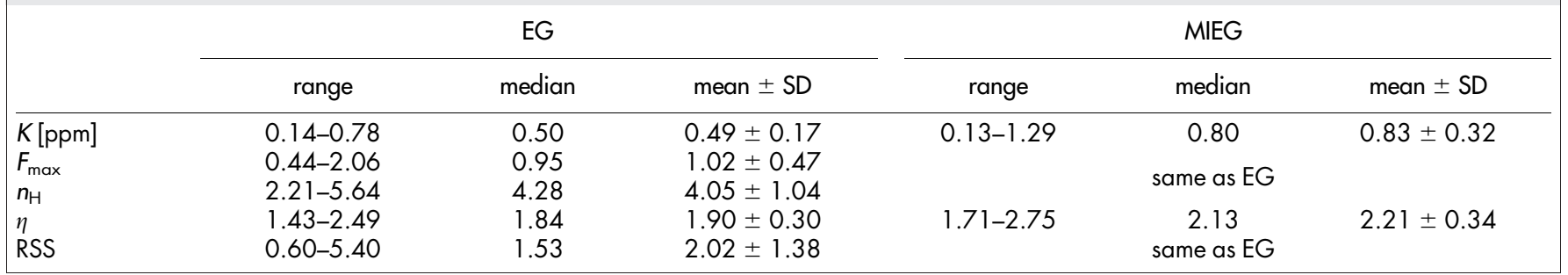


previous observations of hyper- and hypoadditivity in both presynaptic ${ }^{13}$ and postsynaptic ${ }^{7,43}$ responses to odorant mixtures in overlap glomeruli. We formulated a new competitive agonist model that yielded good fits to dose-response relations of overlap glomeruli. The mixture interactions were an inherent property of the non-linear, sigmoid dose-response curve describing saturation of receptor binding and activation, and cooperativity and saturation of olfactory transduction. Our modeling and analysis differs from previous studies in that we separated initial odorant binding and receptor activation from cooperative downstream transduction steps to obtain a self consistent model of mixture interactions. We did not model interglomerular presynaptic inhibition mediated by $\mathrm{GABA}_{\mathrm{B}}$ receptors, which could potentially alter glomerular input patterns at the level of OSN terminals ${ }^{44}$. However, these interactions may be weak ${ }^{45}$, and may not be apparent in highly overlapped glomerular representations.

Antagonism has been reported between EG and MIEG, or other EG derivatives, in the olfactory receptor $\mathrm{mOR}-\mathrm{EG}^{20}$. Oka et al. $(2004)^{21}$ showed that mOR-EG, expressed either heterologously in HEK 293 cells or natively in dissociated OSNs, mediated excitatory responses to EG that were antagonized by MIEG, although MIEG itself does not activate the receptor. Transgenic labeling of mOR-EG expressing OSNs with green fluorescent protein (GFP) revealed that this receptor maps to a glomerulus on the dorsal olfactory bulb ${ }^{30}$. However, we were not able to identify a dorsal glomerulus activated only by EG, and antagonized by MIEG. There were few non-overlap glomeruli in EG and MIEG activation maps (Fig. 1), and none showed antagonistic interactions. Our analysis therefore focused on glomeruli for which both EG and MIEG acted as agonists. We did not have a GFP tag to identify the mOR-EG glomerulus in $\mathrm{spH}$ mice, and its location along the anterolateral edge of the dorsal bulb ${ }^{30}$ may have been overlooked in our imaging preparations which did not completely expose the bulb by removing the skull. Antagonism between EG and MIEG can also occur in other receptors. A calcium imaging analysis of a random sample of 95 EG-responsive OSNs isolated from the mouse found that $21 \%$ were antagonized by MIEG, and only $16 \%$ responded to both EG and MIEG ${ }^{30}$. These percentages suggest a lower overlap of glomerular response maps and more frequent antagonism, in contrast to our findings. However, it is unclear if the analyzed OSNs represented an unbiased sample of cells projecting to the area of olfactory bulb examined in our imaging experiments. The comparison could also be skewed because in vitro OSN responses to odorants delivered directly to receptors can differ from in vivo glomerular responses ${ }^{30}$. Another potential difference may be an alteration of odorant response profiles for receptors in $\mathrm{spH}$ transgenic mice. The $\mathrm{spH}$ construct replaces the coding sequence for olfactory marker protein (OMP), which apparently plays a role in refinement of olfactory receptor tuning during development ${ }^{46}$. We did not detect gross differences between EG and MIEG glomerular maps in mice that were homozygous (with OMP knocked out) and heterozygous (with 50\% OMP expression) for the $\mathrm{spH}$ gene. However, we cannot rule out the possibility that the incidence of antagonism was decreased by a broader tuning of receptors associated with reduction or loss of OMP expression.

Differences between in vitro and in vivo responses of olfactory receptors may be a consequence of biotransformation of odorants by the olfactory mucosa. For example, enzymes in olfactory mucus can break down acetyl isoeugenol (AIEG) into isoeugenol (IEG) during in vivo stimulation ${ }^{47}$. This raises the question of whether the apparently competitive interactions between EG and MIEG reflect enzymatic break down of MIEG to EG prior to receptor binding. Such conversion would also conceal antagonism of EG responses by MIEG. We consider this scenario unlikely because such a conversion would entail several reaction steps, and because non-overlapping glomeruli are detectable in the odor maps (e.g. Fig. 1; see also Ref. 30).
The good fits of our competitive agonist model to EG-MIEG mixture interactions supports the hypothesis that these structurally similar molecules compete and activate receptors at the same binding sites. Although we did not encounter specific examples of competitive antagonism, our model does include this as a specific case in which one ligand binds but fails to activate the receptor. Thus, the model may be generally useful for describing a wide range of competitive mixture interactions. Non-competitive interactions may be more likely between odorants with widely differing molecular structures that could bind to different sites on the receptor and influence each other through allosteric mechanisms. A recent survey of rat OSN responses to a diverse panel of odorants inferred that noncompetitive interactions occur frequently, in ca. $50 \%$ of binary mixtures $^{27}$. One sign of non-competitive interaction was the failure of pure odorant dose-response curves to bracket the dose-response curves of constant ratio binary mixtures. Bracketing and smooth transition between dose-response curves is a property of competitive models, and we did not see any violations of this behavior in our data sets. Allosteric interactions may exist even for odorant pairs that act as competitive agonists at the same receptor site, if either odorant also modulates the receptor through other mechanisms. Such effects might be modeled, for example by allowing receptor activation and efficiency constants $(K, \eta)$ to vary with odorant concentrations. The relative contribution of competitive vs. non-competitive mixture interactions to odor coding is an important issue that warrants further investigation. The frequency of non-competitive interactions seen in the rat OSN study may need re-evaluation because the model used to analyze that data does not describe suprathreshold mixture interactions arising from cooperativity of olfactory transduction (Supplementary Information).

In our model of mixture interaction, we implicitly assumed that all receptors relaying input to a given glomerulus are stimulated by odorants at fixed concentrations. However, it has long been thought that a key step in mixture coding is the separation of mixture components by differential adsorption in the olfactory epithelium by a process similar to gas chromatography ${ }^{9,48-50}$. Since each glomerulus receives receptor inputs from an extended zone of epithelium, receptors at different positions in adsorption gradients might be exposed to different ratios of mixture components. Physical separation of odorants will reduce nonlinear mixture interaction, and increase linear additivity of component responses. However, our nonlinear model provided a good data fit that was not improved by a simple model of odorant separation (Supplementary Information). Chromatographic effects could be small for EG and MIEG, which have similar hydrophobicity. Our study focused on the dorsal olfactory bulb which is innervated by receptors lining the central canal of the nasal cavity ${ }^{51}$. This domain is located upstream in the airflow pathway where it captures more hydrophilic odorants, and there may be little differential adsorption of more hydrophobic odorants such as EG and MIEG. Chromatographic separation could have greater impact on mixture responses of glomeruli which combine inputs from upstream sites with inputs located further downstream. However, such glomeruli may be rare because olfactory receptors tend to be expressed in horizontal zones roughly orthogonal to the airflow axis $^{52}$. Peripheral olfactory anatomy appears to be configured to avoid chromatographic 'linearization' of glomerular mixture responses. This may be advantageous for simplifying mixture coding and reducing the computational tasks of central olfactory circuits.

The importance of peripheral mixture interactions for day-to-day olfaction of humans and animals is still not very well understood. Natural odorant stimuli can contain complex mixtures of many different odorants, and one might suspect that mixture interactions would be common. However, studies show that glomerular representations of natural odorant mixtures can vary widely for different types of stimuli. In some cases, glomerular maps are sparse, which should reduce overlap of component responses and lead to less 
interaction ${ }^{53,54}$; in other cases, maps are dense, which should increase the chances of overlap and interaction ${ }^{55,56}$. In animals, odorant stimuli encountered in natural habitats might be composed of dissimilar compounds at lower concentrations that may be sparsely coded with less mixture interaction. In humans, exposure to mixtures of similar chemicals at higher concentrations might be more frequent in modern cultural environments dominated by artificial synthetic chemistry. The design of fragrance products and odor masking agents may benefit from a better understanding of mixture interactions.

\section{Methods}

All experiments were approved by the Institutional Animal Care and Use Committee at the Monell Chemical Senses Center.

Animal preparation. Imaging data were acquired from adult male and female mice (12-16 weeks postnatal) belonging to two strains: B6;129P2-Omp $p^{\text {tm2(spH)Mom}} / \mathrm{J} \mathrm{x} \mathrm{B6}$ 129 (heterozygous OMP-spH); and B6;129P2-Omp $p^{\text {tm2(spH)Mom } / J ~(h o m o z y g o u s ~ O M P-~}$ $\mathrm{spH})$. Colonies established from breeder pairs obtained from the Jackson Laboratory (Bar Harbor, ME) were maintained in a temperature-, humidity- and light cyclecontrolled facility with ad libitum access to food and water. Prior to each imaging session, mice were anesthetized (ketamine, $90 \mathrm{mg} / \mathrm{kg}, 1 / 2$ dosage i.p.; Xylazine, $10 \mathrm{mg} / \mathrm{kg}, 1 / 2$ dosage i.p.; sodium pentobarbital, $50 \mathrm{mg} / \mathrm{kg}, 1 / 2$ dosage i.p.), body temperature regulated at $37^{\circ} \mathrm{C}$ with a heating pad and rectal probe (TCAT-2LV, Physitemp Instruments, Clifton, NJ), and eyes moistened with ophthalmic ointment. Animals were maintained under deep anesthesia (sodium pentobarbital, 1/3 dosage i.p., every $30 \mathrm{~min}$ ) administered through a subdiaphragmatic cannula. Respiration was monitored by a piezoelectric chest strap (Kent Scientific), and maintained at $2-3 \mathrm{~Hz}$ by anesthetic delivery. Atropine sulfate $(5 \mathrm{mg} / \mathrm{kg}$, s.c.) was administered to reduce bronchial secretion, and dexamethasone $(0.07 \mathrm{mg} / \mathrm{kg}$, s.c.) injected near the surgical site to control inflammation. High plane of anesthesia was confirmed by lack of tail pinch reflex. The skull over the OB was exposed by depilation and midline incision of the scalp under local anesthesia (bupivacaine, $8 \mathrm{mg} / \mathrm{kg}$, s.c.). Connective tissue was removed, the surface wiped dry, and a custom head plate was attached with dental cement to immobilize the head. Bone over the $\mathrm{OB}$ was gradually thinned to transparency with a $2-2.5 \mathrm{~mm}$ drill bit (4000 r.p.m.) cooled with saline, and

$\sim 10-30 \mu \mathrm{m}$ thick window created by scraping with a dental micro-blade. A layer of saline was held over the window by a Vaseline ring and glass coverslip). In reciprocal experiments, mice were held over 2 days after recovery from the first imaging session, with analgesic (buprenorphine $0.05-0.1 \mathrm{mg} / \mathrm{kg}$, s.c.) administered every $6-12 \mathrm{~h}$.

Odorant stimulation. Freshly prepared solutions of neat odorant (eugenol (EG), 99\%; methyl isoeugenol MIEG), $\geq 98 \%$, Sigma-Aldrich) were diluted to various concentrations in mineral oil and placed in air-tight bottles connected to a custombuilt olfactometer controlled by LabVIEW software (National Instruments, Austin, TX). Under control conditions, a constant flow of clean air $(1500 \mathrm{ml} / \mathrm{min})$ passed through a bottle of mineral oil (blank stimulus), and exited a nozzle directed at the mouse external nares. During stimulation, a solenoid valve switched the flow from blank to a $\mathrm{T}$-junction splitting the airstream to either of two test odorants singly, or a binary mixture. To avoid headspace depletion due to low odorant vapor pressure, we used multiple single-shot stimulus bottles pre-warmed to $45^{\circ} \mathrm{C}$ to provide more consistent vapor concentrations. A photoionization detector (PID) (ppbRAE3000, RAE Systems, San Jose, CA) measured concentrations of odorant at the exit nozzle. These concentrations were lower than headspace concentrations due to air dilution of vapor passing through olfactometer lines and valves. Dose-response relations for single odorants and binary mixtures were expressed in terms of nominal PID readings calibrated with isobutylene standard, expressed in parts per million (ppm) by volume (i.e. molar concentration). In analysis and model fitting, we used PID readings as direct proportional measures of concentration. Correction factors for EG and MIEG were not determined, so arbitrary scale factors were associated with concentration units of each odorant. To minimize spurious effects of odorant adsorption to olfactometer parts, we checked that baseline PID readings were restored at the end of inter-stimulus intervals, and blank (mineral oil) stimulus did not evoke detectable $\mathrm{spH}$ responses; flow lines were washed and plastic tubing replaced after each experiment.

Odor stimuli were delivered at 2 min intervals in three repeated trials. For each trial, imaging data were acquired over a $20 \mathrm{~s}$ period with a $3-5$ s pre-stimulus segment preceding a $10 \mathrm{~s}$ odorant (or blank) stimulus pulse. Experiments were performed in 2 stages: (i) the dose-response relation of a test odorant (EG or MIEG) was established at 5 stimulus concentrations $(0.1,1,10,50 \% \mathrm{v} / \mathrm{v}$ liquid dilution, neat), in shuffled ascending order (higher concentration at end of session) to minimize hysteresis and long term adaptation or desensitization; (ii) responses to binary mixtures were recorded by adding the second odorant at 3 concentrations $(10,30,70 \% \mathrm{v} / \mathrm{v}$ liquid dilution), mixed with test odorant in vapor phase. Test stimuli consisting of the second odorant alone $(10,30,70 \% \mathrm{v} / \mathrm{v}$ liquid dilution) were interleaved with the trials as controls (semi-random shuffling, ascending order). Testing highest concentrations near the end of each experiment minimized the impact of desensitization. No significant changes in peak $\mathrm{spH}$ fluorescence of high concentration stimuli over the $\sim 3-4$ h experimental period were detected. Each mouse was tested with either of two reciprocal protocols: EG (test), MIEG (second odorant); or MIEG (test), EG (second odorant) on different days.

Image acquisition and analysis. The dorsal $\mathrm{OB}$ of $\mathrm{OMP}-\mathrm{spH}$ mice was viewed under an Olympus BX50WI microscope equipped with a $4 \times$ XLFluor objective (NA 0.28). Glomerular fluorescence was excited by light from a cyan LED source (LEDC9, Thorlabs, Newton, NJ), directed through a long pass filter set: $480 \mathrm{~nm}$ excitation, $505 \mathrm{~nm}$ dichroic, $510 \mathrm{~nm}$ emission (41012 FITC/RSGFP LP, Chroma Technology, Bellows Falls, VT). Images were acquired by a Photometrics Cascade II 512B EMCCD camera (Optical Apparatus, Ardmore, PA) under control of ImagePro 6.0 software (Media Cybernetics, Bethesda, MD), with a LabVIEW program synchronizing the camera and olfactometer. Image sequences $(20 \mathrm{~s})$ were acquired at $10 \mathrm{~Hz}, 16$-bits, with $2 \times 2$ binning (resolution $256 \times 256$ pixels). This was sufficient to capture the rising phase and peak of responses. We did not routinely record recovery which lasted ca. $1 \mathrm{~min}$.

Image processing was implemented by algorithms and custom macros in ImagePro 6.0. To isolate stimulus-evoked glomerular activity, we first corrected photobleaching in each trial using paired blank trials. Signal-to-noise ratio was then increased by averaging across trials and time binning. Image sequences were replayed to observe fluorescence changes across the field of view, allowing identification and exclusion of false positive responses. Glomerular maps of fluorescence increase were computed by subtracting pre-stimulus baseline from peak fluorescence. Maps were spatially calibrated, filtered by a Gaussian kernel (size 2), rendered in pseudocolor, and thresholded at intensity levels 1-3 standard deviations (SD) over mean intensity. Putative glomeruli were detected as localized regions of interest (ROIs) with high signal, and their centers of mass and diameters calculated. ROIs had diameters of 36-133 $\mu \mathrm{m}$, consistent with previously reported $\mathrm{spH}$ foci ${ }^{29}$. For each ROI, fluorescence was tracked over time in raw, unprocessed images from paired odorant and blank trials. We rejected as spurious ROIs correlated with preparation movement, and ROIs without clear odorant dose-response relations; remaining ROIs were classified as glomeruli.

Overlap between glomerular maps was quantified by colocalization analysis. A binary threshold was set interactively to include all putative glomeruli, and Pearson's correlation coefficient:

$$
\begin{aligned}
& \sum\left(\operatorname{Odor}_{i}-\operatorname{Odor}_{\text {ave }}\right) .\left(\operatorname{Odor} 2_{i}-\text { Odor }_{\text {ave }}\right) \\
& r=\frac{i}{\sqrt{\sum_{i}\left(\text { Odor } 1_{i}-\text { Odor } 1_{\text {ave }}\right)^{2} \cdot \sum_{j}\left(\text { Odor } 2_{j}-\text { Odor } 2_{\text {ave }}\right)^{2}}}
\end{aligned}
$$

was calculated between pairs of maps from the same animal and experiment; Odor $\mathrm{K}_{i}$ (or Odor $\mathrm{K}_{\text {ave }}$ ) is the signal intensity at pixel $i$ (or the average intensity) for the response map of odorant $\mathrm{K}$.

Odorant dose-response and mixture analysis. We selected a set of $n=18$ glomeruli from 4 mice for analysis of response kinetics, and a subset of these $(n=15)$ for fitting models to dose-response data. Variability in glomerular positions prevented us from identifying glomeruli across different mice belonging to the same olfactory receptors, so our analyses may represent the properties of a smaller set of receptors. For each ROI, we applied photobleaching correction to the mean fluorescence time course, and computed relative fluorescence increase, $\Delta \mathrm{F} / \mathrm{F}_{0}(t)$, where $\Delta \mathrm{F}$ is the difference between response amplitude at time $t$ and $\mathrm{F}_{0}$, the pre-stimulus baseline fluorescence signal (estimated by averaging $5-10$ frames). The rising phase and peak of each $\Delta \mathrm{F} / \mathrm{F}_{0}$ response was quantified by empirical fits to an 'alpha' function: $y=A$. $\left(t-t_{0}\right)^{v}$. exp $\left(-\left(t-t_{0}\right) / \tau\right)$, with parameters $A$ (amplitude), $v$ (exponent), $\tau$ (decay time), and $x_{0}$ (response start time) (Fig. 2a). We then estimated the peak amplitude (i.e. odorant response) as: $F=y_{\max }=A .(v \tau)^{v}$. $\mathrm{e}^{-v}$; and time-to-peak as: $t_{\max }-t_{0}=v \tau$. Although a secondary peak occasionally appeared in responses, we only fitted alpha functions to match the first peak.

For single odorants, dose-response relations were fitted to a 1D modified Hill equation derived from a model of non-cooperative receptor binding, single-step receptor activation, and cooperative activation of the downstream transduction cascade (Eqn. (1)). Sets of fitted parameters for EG and MIEG were compared using paired, two-tailed t-tests, after confirming normality by a Kolmogorov-Smirnov $(\mathrm{K}-\mathrm{S})$ test $(\mathrm{P}<0.05)$. For binary mixtures, deviations from additivity were detected by applying pair-wise, one-tailed K-S tests to delineate hypoadditive and hyperadditive responses for each glomerulus and concentration pair $(\mathrm{P}<0.05)$. Distributions of summed responses were obtained by convolution of distributions of responses to single odorants across 3 trials. Binary mixture dose-response relations were fitted to a $2 \mathrm{D}$ function of paired odorant concentrations $(U, V)=([\mathrm{EG}],[\mathrm{MIEG}])$ derived from a model of non-cooperative single-site competitive binding of two odorants, singlestep receptor activation, and cooperative activation of the transduction cascade (Eqn (2) in text). Model parameter starting values for 2D fitting iterations were values estimated from 1D fits to single odorant dose-response measurements. Model output was evaluated by comparing residual sums-of-squares (RSS) from best fit curves. Fitting and statistical analyses were performed by iterative nonlinear least squares routines in Origin 7.0 (OriginLab, Northampton, MA) and by Statistica 10 (Stat Soft, Inc., Tulsa, OK). To validate fitting routines, we generated mock data sets from known input parameter values (matching values obtained from 4 different test glomeruli) by calculation from model equations, and added random Gaussian noise to simulate experimental error. Noise distributions were selected to mimic observed dose-dependent variance in measured dose-response relations (CV ranging over $90 \%-4 \%$ from low to high concentrations). The fitting routines were then applied to 
simulated data to estimate known input parameters. In every trial, fitting could reliably recover values of the model parameters that were not significantly different from the input values (Tables ST1, ST2).

In homozygous OMP-spH mice, expression of olfactory marker protein (OMP) is disrupted, which can cause olfactory deficits ${ }^{46,57,58}$. However, we did not find significant differences in magnitudes of $\mathrm{spH}$ fluorescence responses of glomeruli between homozygotes and heterozygotes, so we pooled and analyzed data sets from both.

1. Cain, W. S. Odor intensity: mixtures and masking. Chemical Senses and Flavor 1, 339-352 (1975).

2. Cain, W. S., Schiet, F. T., Olsson, M. J. \& de Wijk, R. A. Comparison of models of odor interaction. Chem. Senses 20, 625-637 (1995).

3. Laing, D. G., Panhuber, H. \& Slotnick, B. M. Odor masking in the rat. Physiol Behav. 45, 689-694 (1989)

4. Laing, D. G., Panhuber, H., Willcox, M. E. \& Pittman, E. A. Quality and intensity of binary odor mixtures. Physiol Behav. 33, 309-319 (1984).

5. Jones, F. N. \& Woskow, M. H. On the intensity of odor mixtures. Ann. N. Y. Acad. Sci. 116, 484-494 (1964).

6. Kay, L. M., Crk, T. \& Thorngate, J. A redefinition of odor mixture quality. Behav. Neurosci. 119, 726-733 (2005).

7. Grossman, K. J., Mallik, A. K., Ross, J., Kay, L. M. \& Issa, N. P. Glomerular activation patterns and the perception of odor mixtures. Eur. J. Neurosci. 27, 2676-2685 (2008).

8. Wiltrout, C., Dogra, S. \& Linster, C. Configurational and nonconfigurational interactions between odorants in binary mixtures. Behav. Neurosci. 117, 236-245 (2003).

9. Bell, G. A., Laing, D. G. \& Panhuber, H. Odour mixture suppression: evidence for a peripheral mechanism in human and rat. Brain Res 426, 8-18 (1987).

10. Duchamp-Viret, P., Duchamp, A. \& Chaput, M. A. Single olfactory sensory neurons simultaneously integrate the components of an odour mixture. Eur. J. Neurosci. 18, 2690-2696 (2003).

11. Chaput, M. et al. Interactions of odorants with olfactory receptors and receptor neurons match the perceptual dynamics observed for woody and fruity odorant mixtures. Behav. Neurosci. 35, 584-597 (2012).

12. Linster, C. \& Cleland, T. A. Configurational and elemental odor mixture perception can arise from local inhibition. J. Comput. Neurosci. 16, 39-47 (2004).

13. Tabor, R., Yaksi, E., Weislogel, J. M. \& Friedrich, R. W. Processing of odor mixtures in the zebrafish olfactory bulb. J. Neurosci. 24, 6611-6620 (2004).

14. Takahashi, Y. K., Nagayama, S. \& Mori, K. Detection and masking of spoiled food smells by odor maps in the olfactory bulb. J. Neurosci. 24, 8690-8694 (2004).

15. Malnic, B., Hirono, J., Sato, T. \& Buck, L. B. Combinatorial receptor codes for odors. Cell 96, 713-723 (1999).

16. Nara, K., Saraiva, L. R., Ye, X. \& Buck, L. B. A large-scale analysis of odor coding in the olfactory epithelium. J. Neurosci. 31, 9179-9191 (2011).

17. Price, S. Effects of odorant mixtures on olfactory receptor cells. Ann. N. Y. Acad. Sci. 510, 55-60 (1987).

18. Sanz, G., Schlegel, C., Pernollet, J. C. \& Briand, L. Comparison of odorant specificity of two human olfactory receptors from different phylogenetic classes and evidence for antagonism. Chem. Senses 30, 69-80 (2005).

19. Araneda, R. C., Peterlin, Z., Zhang, X., Chesler, A. \& Firestein, S. A pharmacological profile of the aldehyde receptor repertoire in rat olfactory epithelium. J. Physiol 555, 743-756 (2004).

20. Oka, Y., Nakamura, A., Watanabe, H. \& Touhara, K. An odorant derivative as an antagonist for an olfactory receptor. Chem. Senses 29, 815-822 (2004).

21. Oka, Y., Omura, M., Kataoka, H. \& Touhara, K. Olfactory receptor antagonism between odorants. EMBO J. 23, 120-126 (2004).

22. Ukhanov, K., Brunert, D., Corey, E. A. \& Ache, B. W. Phosphoinositide 3-Kinasedependent antagonism in mammalian olfactory receptor neurons. J. Neurosci. 31, 273-280 (2011).

23. Ukhanov, K., Corey, E. A., Brunert, D., Klasen, K. \& Ache, B. W. Inhibitory odorant signaling in Mammalian olfactory receptor neurons. J. Neurophysiol. 103, 1114-1122 (2010)

24. Takeuchi, H., Ishida, H., Hikichi, S. \& Kurahashi, T. Mechanism of olfactory masking in the sensory cilia. J. Gen. Physiol 133, 583-601 (2009).

25. Kawai, F. Odorants suppress T- and L-type Ca2+ currents in olfactory receptor cells by shifting their inactivation curves to a negative voltage. Neurosci. Res. 35 , 253-263 (1999).

26. Kawai, F. Odorant suppression of delayed rectifier potassium current in newt olfactory receptor cells. Neurosci. Lett. 269, 45-48 (1999).

27. Rospars, J. P., Lansky, P., Chaput, M. \& Duchamp-Viret, P. Competitive and noncompetitive odorant interactions in the early neural coding of odorant mixtures. J. Neurosci. 28, 2659-2666 (2008).

28. Mombaerts, P. et al. Visualizing an olfactory sensory map. Cell 87, 675-686 (1996).

29. Bozza, T., McGann, J. P., Mombaerts, P. \& Wachowiak, M. In vivo imaging of neuronal activity by targeted expression of a genetically encoded probe in the mouse. Neuron 42, 9-21 (2004).

30. Oka, Y. et al. Odorant receptor map in the mouse olfactory bulb: in vivo sensitivity and specificity of receptor-defined glomeruli. Neuron 52, 857-869 (2006).
31. Kobayakawa, K. et al. Innate versus learned odour processing in the mouse olfactory bulb. Nature 450, 503-508 (2007).

32. Schaefer, M. L., Finger, T. E. \& Restrepo, D. Variability of position of the P2 glomerulus within a map of the mouse olfactory bulb. J. Comp Neurol. 436, 351-362 (2001)

33. Frederick, D. E., Barlas, L., Ievins, A. \& Kay, L. M. A critical test of the overlap hypothesis for odor mixture perception. Behav. Neurosci. 123, 430-437 (2009).

34. Wachowiak, M. et al. Inhibition of olfactory receptor neuron input to olfactory bulb glomeruli mediated by suppression of presynaptic calcium influx. J. Neurophysiol. 94, 2700-2712 (2005).

35. Wachowiak, M. \& Cohen, L. B. Representation of odorants by receptor neuron input to the mouse olfactory bulb. Neuron 32, 723-735 (2001).

36. Vermeulen, A., Lansky, P., Tuckwell, H. \& Rospars, J. P. Coding of odour intensity in a sensory neuron. Biosystems 40, 203-210 (1997).

37. Bakalyar, H. A. \& Reed, R. R. Identification of a specialized adenylyl cyclase that may mediate odorant detection. Science 250, 1403-1406 (1990).

38. Jones, D. T. \& Reed, R. R. Golf: an olfactory neuron specific-G protein involved in odorant signal transduction. Science 244, 790-795 (1989).

39. Sklar, P. B., Anholt, R. R. \& Snyder, S. H. The odorant-sensitive adenylate cyclase of olfactory receptor cells. Differential stimulation by distinct classes of odorants. J Biol Chem 261, 15538-15543 (1986)

40. Lowe, G. \& Gold, G. H. Nonlinear amplification by calcium-dependent chloride channels in olfactory receptor cells. Nature 366, 283-286 (1993).

41. Cleland, T. A. \& Linster, C. Concentration tuning mediated by spare receptor capacity in olfactory sensory neurons: A theoretical study. Neural Comput. 11, 1673-1690 (1999).

42. Takeuchi, H. \& Kurahashi, T. Mechanism of signal amplification in the olfactory sensory cilia. J. Neurosci. 25, 11084-11091 (2005).

43. Fletcher, M. L. Analytical processing of binary mixture information by olfactory bulb glomeruli. PLoS. One. 6, e29360 (2011).

44. Vucinic, D., Cohen, L. B. \& Kosmidis, E. K. Interglomerular center-surround inhibition shapes odorant-evoked input to the mouse olfactory bulb in vivo. J. Neurophysiol. 95, 1881-1887 (2006).

45. McGann, J. P. et al. Odorant representations are modulated by intra- but not interglomerular presynaptic inhibition of olfactory sensory neurons. Neuron $\mathbf{4 8}$ 1039-1053 (2005).

46. Lee, A. C., He, J. \& Ma, M. Olfactory marker protein is critical for functional maturation of olfactory sensory neurons and development of mother preference. J. Neurosci. 31, 2974-2982 (2011).

47. Nagashima, A. \& Touhara, K. Enzymatic conversion of odorants in nasal mucus affects olfactory glomerular activation patterns and odor perception. J. Neurosci. 30, 16391-16398 (2010).

48. Mozell, M. M. \& Jagodowicz, M. Chromatographic separation of odorants by the nose: retention times measured across in vivo olfactory mucosa. Science 181, 1247-1249 (1973).

49. Mozell, M. M. Evidence for sorption as a mechanism of the olfactory analysis of vapours. Nature 203, 1181-1182 (1964)

50. Laing, D. G. Coding of chemosensory stimulus mixtures. Ann. N. Y. Acad. Sci. 510, 61-66 (1987).

51. Miyamichi, K., Serizawa, S., Kimura, H. M. \& Sakano, H. Continuous and overlapping expression domains of odorant receptor genes in the olfactory epithelium determine the dorsal/ventral positioning of glomeruli in the olfactory bulb. J. Neurosci. 25, 3586-3592 (2005).

52. Schoenfeld, T. A. \& Cleland, T. A. The anatomical logic of smell. Trends Neurosci. 28, 620-627 (2005)

53. Schaefer, M. L., Yamazaki, K., Osada, K., Restrepo, D. \& Beauchamp, G. K. Olfactory fingerprints for major histocompatibility complex-determined body odors II: relationship among odor maps, genetics, odor composition, and behavior. J. Neurosci. 22, 9513-9521 (2002).

54. Lin, D. Y., Shea, S. D. \& Katz, L. C. Representation of natural stimuli in the rodent main olfactory bulb. Neuron 50, 937-949 (2006)

55. Johnson, B. A., Ong, J. \& Leon, M. Glomerular activity patterns evoked by natural odor objects in the rat olfactory bulb are related to patterns evoked by major odorant components. J. Comp Neurol. 518, 1542-1555 (2010).

56. Vincis, R., Gschwend, O., Bhaukaurally, K., Beroud, J. \& Carleton, A. Dense representation of natural odorants in the mouse olfactory bulb. Nat. Neurosci. 15, 537-539 (2012)

57. Reisert, J., Yau, K. W. \& Margolis, F. L. Olfactory marker protein modulates the cAMP kinetics of the odour-induced response in cilia of mouse olfactory receptor neurons. J. Physiol 585, 731-740 (2007).

58. Youngentob, S. L., Pyrski, M. M. \& Margolis, F. L. Adenoviral vector-mediated rescue of the OMP-null behavioral phenotype: enhancement of odorant threshold sensitivity. Behav. Neurosci. 118, 636-642 (2004).

\section{Acknowledgements}

This work was supported by a U.S. Public Health Service Grant from the National Institute of Deafness and other Communication Disorders, 5R01DC004208, and an industry grant from Japan Tobacco, Inc. We are grateful to Dr Johannes Reisert and Dr Joel Mainland for critical comments on the manuscript, and to Jinghua Chai for technical support. 


\section{Author contributions}

Both G.C. and G.L. conceived and designed the experiments and model, and wrote the manuscript; G.C. performed the experiments and analyzed the data.

\section{Additional information}

Supplementary information accompanies this paper at http://www.nature.com/ scientificreports
Competing financial interests: This work was supported in part by an industry grant from Japan Tobacco, Inc.

License: This work is licensed under a Creative Commons

Attribution-NonCommercial-NoDerivs 3.0 Unported License. To view a copy of this license, visit http://creativecommons.org/licenses/by-nc-nd/3.0/

How to cite this article: Cruz, G. \& Lowe, G. Neural coding of binary mixtures in a structurally related odorant pair. Sci. Rep. 3, 1220; DOI:10.1038/srep01220 (2013). 